\title{
Biological Synthesis of Silver Nanoparticles and Its Antibacterial Activity
}

Hinal Gandhi* and Shabib Khan

Department of Microbiology, K.J Somaiya College of Science and Commerce, Vidyavihar, Mumbai 400077, Maharashtra, India

\begin{abstract}
The ability of prokaryotic microorganisms to reduce the inorganic metals has opened up an exciting ecofriendly approach towards the development of green nanotechnology. Thus synthesis of metal nanoparticles through biological approach is an important aspect of current nanotechnology. In this study, silver nanoparticles have been successfully synthesized by Escherichia coli, in which exposure of supernatant to silver ions resulted in the extracellular reduction of the metal ions and formation of silver nanoparticles. Antimicrobial activity of silver nanoparticles was evaluated for their combined effects with antibiotics against microbes. The antibacterial activities increased in the presence of biologically synthesized silver nanoparticles. The enhancing effect was observed for Bacitracin against Escherichia coli and Salmonella paratyphi B., for Ampicillin against Corynebacterium diphtheriae, for Kanamycin against Klebsiella pneumonia, for gentamycin against Pseudomonas aeruginosa and for Bacitracin, Gentamycin, Erythromycin, Ciprofloxacin against Staphylococcus aureus.
\end{abstract}

Keywords: Green nanotechnology; Silver nanoparticles; Combined effect; Antimicrobial activity

\section{Introduction}

Nanotechnology is an emerging field in the area of interdisciplinary research, especially in biotechnology. The synthesis of silver nanomaterials/nanoparticles is extensively studied by using chemical and physical methods, but the development of reliable technology to produce nanoparticles is an important aspect of nanotechnology [1]. Biological synthesis process provides a wide range of environmentally acceptable methodology, low cost production and minimum time required. At the same time the biologically synthesized silver nanoparticles has many applications such as catalysts in chemical reactions.

Nanotechnology literally means any technology on a nanoscale that has applications in the real world. Nanotechnology encompasses the production and application of physical, chemical, and biological systems at scales ranging from individual atoms or molecules to submicron dimensions, as well as the integration of the resulting nanostructures into larger systems. Nanotechnology is likely to have a profound impact on our economy and society in the early $21^{\text {st }}$ century, comparable to that of semiconductor technology, information technology, or cellular and molecular biology [2]. Science and technology research in nanotechnology promises breakthroughs in areas such as materials and manufacturing, nanoelectronics, medicine and healthcare, energy, biotechnology, information technology, and national security. It is widely felt that nanotechnology will be the next Industrial Revolution.

Several chemical methods have been developed for the synthesis of silver nanoparticles including chemical reduction, aqueous solution chemical reduction, nonaqueous chemical reduction, the template method, electrochemical reduction, ultrasonic-assisted reduction, photo-induced or photo-catalytic reduction, microwave assisted synthesis, irradiation reduction, the microemulsion method, biochemical method etc., but these chemical methods have been reported along with various drawbacks, including the use of toxic solvents, generation of hazardous by-products, and high energy consumption, which pose potential risks to human health and to the environment [3-5]. Currently, there is a growing need to develop an environment friendly nanoparticle synthesis that does not use toxic chemicals in the process of its synthesis. The microbial-mediated biological synthesis of metallic nanoparticles has recently been recognized as a promising source for mining nanomaterials [6]. The microbial recovery of precious metals with the formation of their nanoparticles is a green alternative to the conventional method. Biosynthesis of silver nanoparticles using bacteria, fungi, and plants are already well-documented. However, the exploration of actinomycetes has recently gained interest for the efficient biological synthesis of metallic nanoparticles. The bonding reaction between the antibiotic and nanoparticles enhances the inhibition effect against the test organisms. The antibiotic molecules contain many active groups such as hydroxyl and amide groups, which react easily with nanosilver by chelation, and helps in effective inhibition [7-12].

In the present investigation, we examined extracellular biosynthesis of silver nanoparticles (AgNPs) from the bacterial strain Escherichia coli. The antibacterial activity of synthesized silver nanoparticles coated on the antibiotics was also studied to ensure the contribution of biologically synthesized silver nanoparticles to Nanomedicine [13].

\section{Materials and Methods}

\section{Synthesis of silver nanoparticles using Escherichia coli}

Materials: 24 hours old Escherichia coli culture; Sterile Nutrient broth; Distilled water; $1 \mathrm{mM}$ Silver nitrate

\section{Methods}

Production of biomass: The bacterial strain Escherichia coli was inoculated into sterile nutrient broth for production of biomass. The culture flasks were incubated on an orbital shaker at $27^{\circ} \mathrm{C}$ and agitated at $220 \mathrm{rpm}$. The biomass was harvested after 24 hours of growth and centrifuged at $12000 \mathrm{rpm}$ for 10 minutes. The supernatant material was collected for the further reaction to synthesize nanoparticles.

*Corresponding author: Hinal Gandhi, Department of Microbiology, K.J Somaiya College of Science and Commerce, Vidyavihar, Mumbai 400077, Maharashtra, India, Tel: 02221025919; E-mail: hinalgandhi1309@gmail.com

Received April 05, 2016; Accepted April 14, 2016; Published April 21, 2016

Citation: Gandhi H, Khan S (2016) Biological Synthesis of Silver Nanoparticles and Its Antibacterial Activity. J Nanomed Nanotechnol 7: 366. doi:10.4172/2157 7439.1000366

Copyright: $\odot 2016$ Gandhi H, et al. This is an open-access article distributed under the terms of the Creative Commons Attribution License, which permits unrestricted use, distribution, and reproduction in any medium, provided the original author and source are credited. 
Citation: Gandhi H, Khan S (2016) Biological Synthesis of Silver Nanoparticles and Its Antibacterial Activity. J Nanomed Nanotechnol 7: 366. doi:10.4172/2157-7439.1000366

Page 2 of 3

Synthesis of silver nanoparticles: The bacterial strain Escherichia coli was cultured, to produce the biomass for biosynthesis in nutrient broth. The collected $100 \mathrm{ml}$ supernatant was added into the reaction vessel containing $1 \mathrm{mM}$ of silver nitrate, control was also run containing only silver nitrate in water and blank was also run containing only biomass. All the reactions were carried out in bright condition for 24 hours.

\section{Antibacterial activity of silver nanoparticles coated on antibiotics}

Materials: Sterile Mueller Hinton agar; Silver nanoparticles solution; Antibiotics discs (Bacitracin (10 U); Streptomycin (10 mcg); Gentamycin $(10 \mathrm{mcg})$; Kanamycin $(30 \mathrm{mcg})$; Ampicillin $(10 \mathrm{mcg})$; Ciprofloxacin $(10 \mathrm{mcg})$; Erythromycin $(10 \mathrm{mcg})$ ); Different Gram negative and Gram positive bacterial cultures; Sterile petri plates; Sterile swabs

\section{Method}

The sterile molten Mueller Hinton agar was poured in sterile petriplates, allowed to solidify, then different cultures Escherichia coli, Salmonella paratyphi B., Corynebacterium diphtheria, Klebsiella pneumoniae, Pseudomonas aeruginosa, Staphylococcus aureus were swabbed on different plates and allowed to absorb respectively. After 10 mins different antibiotic discs Bacitracin (10 U), Ampicillin (10 mcg), Kanamycin $(30 \mathrm{mcg})$, Gentamycin $(10 \mathrm{mcg})$, Erythromycin $(10 \mathrm{mcg})$, Ciprofloxacin $(10 \mathrm{mcg})$ were dipped in the silver nanoparticles solution to get coated and placed on the plates which were incubated for 24 hours at $37^{\circ} \mathrm{C}$. Zones of inhibition were observed after incubation.

\section{Results and Discussion}

\section{Synthesis of silver nanoparticles using Escherichia coli}

Silver nanoparticles were biologically synthesized by the culture supernatant Escherichia coli. Color change from yellow to brown in the silver nitrate containing flask indicated the formation of silver nanoparticles, whereas no color change was observed in control and blank flask (Figure 1).

\section{Antibacterial activity of silver nanoparticles coated on antibiotics}

The biologically synthesized AgNPs inhibited different microorganisms. The resulting zones of inhibition formed were mainly due to the destabilization of the outer membrane, collapse of the plasma membrane, and depletion of intracellular ATP by the silver nanoparticles. Escherichia coli, Pseudomonas aeruginosa, Salmonella sp, Staphylococcus aureus. The combination of antibiotics with AgNPs against Gram-positive and Gram-negative bacteria offers a valuable contribution to nanomedicine. The antibacterial activities of Bacitracin, Streptomycin, Gentamycin, Kanamycin, Ampicillin increased in the presence of AgNPs against the test strains. The measured zone of inhibition is shown in Table 1 and Figures 1-7.

\section{Conclusions}

The present study concludes that Escherichia coli can be used as source for synthesis of silver nanoparticles extracellulary. The biosynthetic methods have been recognized as an alternative to chemical and physical synthesis, as this biosynthetic method is economical, ecofriendly, and cost-effective. The present work exhibited an efficient and low-cost biological approach to synthesize the metal nanoparticles and provided helpful insight into the development of new antimicrobial agents with the synergistic enhancement of the antibacterial mechanism

\begin{tabular}{|c|c|c|c|}
\hline \multirow{2}{*}{ Name of organisms } & Antibiotics used & \multicolumn{2}{|c|}{$\begin{array}{c}\text { Zone of inhibition } \\
\text { in mm }\end{array}$} \\
\cline { 2 - 4 } & & Control & Test \\
\hline Escherichia coli & Bacitracin $(10 \mathrm{U})$ & 00 & 22 \\
\hline Salmonella paratyphiB & Bacitracin $(10 \mathrm{U})$ & 00 & 18 \\
\hline Corynebacterium diphtheriae & Ampicillin $(10 \mathrm{mcg})$ & 00 & 14 \\
\hline Klebsiella pneumoniae & Kanamycin $(30 \mathrm{mcg})$ & 19 & 30 \\
\hline \multirow{2}{*}{ Pseudomonas aeruginosa } & Gentamycin $(10 \mathrm{mcg})$ & 18 & 34 \\
\hline \multirow{2}{*}{ Staphylococcus aureus } & Bacitracin $(10 \mathrm{U})$ & 00 & 12 \\
\cline { 2 - 4 } & Gentamycin $(10 \mathrm{mcg})$ & 14 & 29 \\
\cline { 2 - 4 } & Erythromycin $(10 \mathrm{mcg})$ & 19 & 32 \\
\cline { 2 - 4 } & Ciprofloxacin $(10 \mathrm{mcg})$ & 21 & 30 \\
\hline
\end{tabular}

Table 1: Antibacterial activity of silver nanoparticles coated on antibiotics.

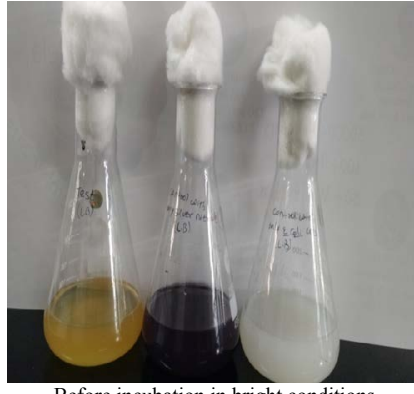

Before incubation in bright conditions

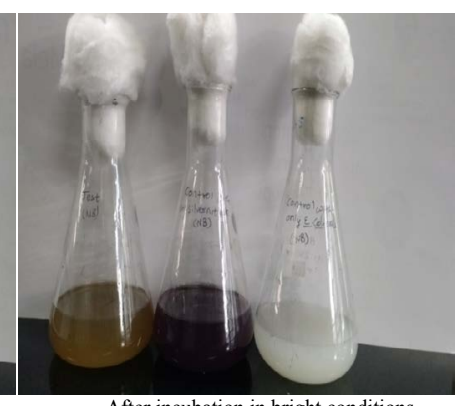

After incubation in bright conditions.
Figure 1: Color change from yellow to brown after incubation indicates presence of silver nanoparticles.

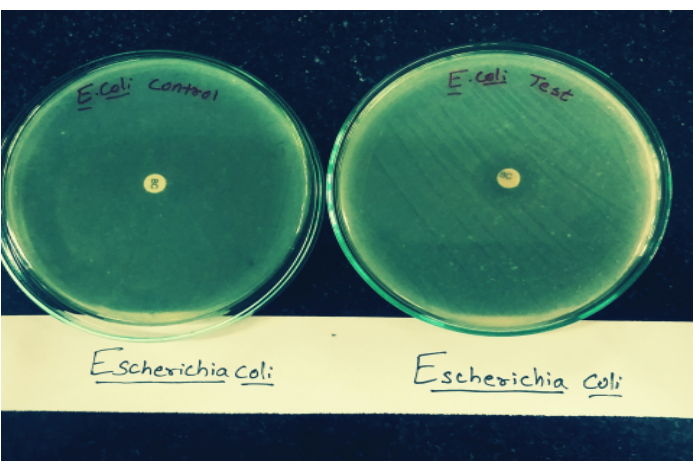

Figure 2: Antibacterial activity of biologically synthesized $1 \mathrm{mM}$ silver nanoparticles coated on antibiotic bacitracin (10 U) against Escherichia coli.

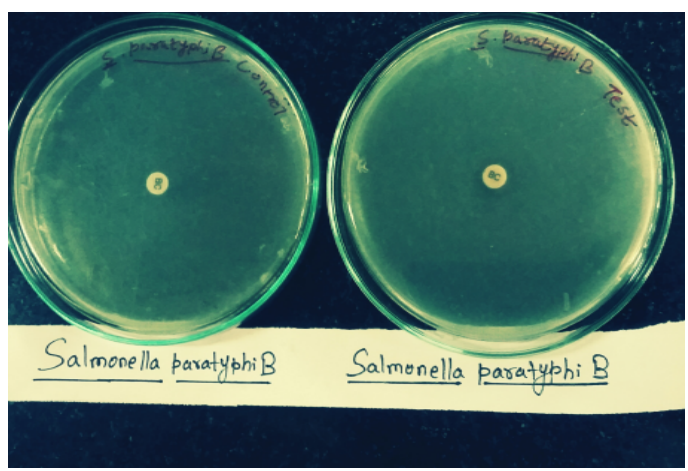

Figure 3: Antibacterial activity of biologically synthesized $1 \mathrm{mM}$ silver nanoparticles coated on antibiotic bacitracin (10 U) against Salmonella paratyphi B. 
Citation: Gandhi H, Khan S (2016) Biological Synthesis of Silver Nanoparticles and Its Antibacterial Activity. J Nanomed Nanotechnol 7: 366. doi:10.4172/2157-7439.1000366

Page 3 of 3

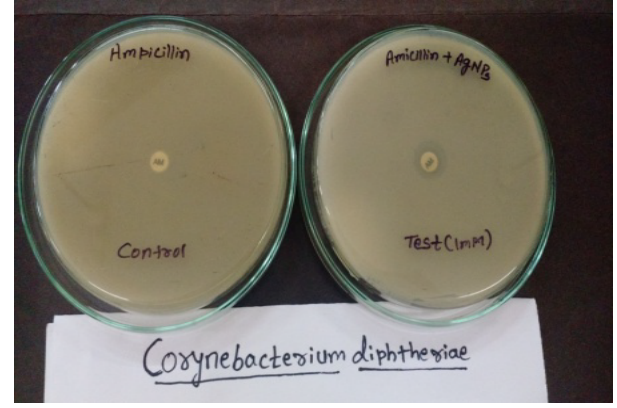

Figure 4: Antibacterial activity of biologically synthesized $1 \mathrm{mM}$ silver nanoparticles coated on antibiotic ampicillin $(10 \mathrm{mcg})$ against Corynebacterium diphtheria.

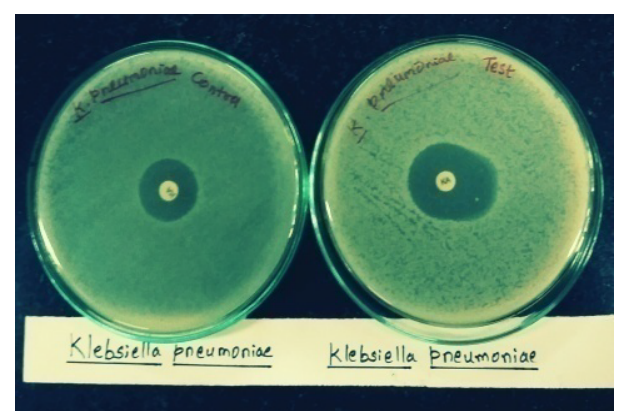

Figure 5: Antibacterial activity of biologically synthesized $1 \mathrm{mM}$ silver nanoparticles coated on antibiotic kanamycin $(30 \mathrm{mcg})$ against Klebsiella pneumonia.

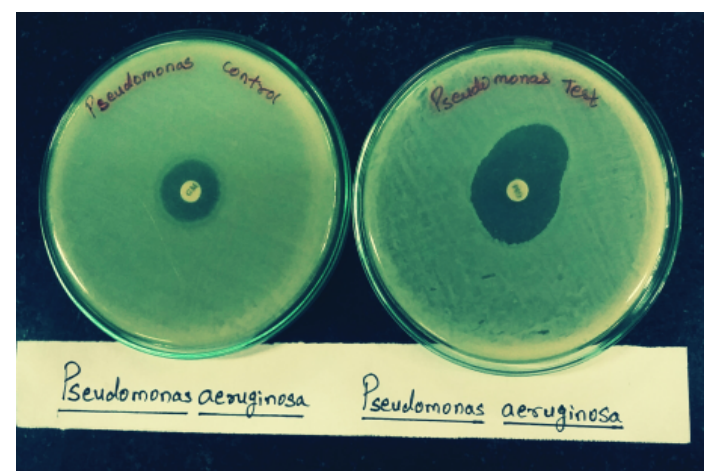

Figure 6: Antibacterial activity of biologically synthesized $1 \mathrm{mM}$ silver nanoparticles coated on antibiotic gentamycin $(10 \mathrm{mcg})$ against $P$ seudomonas aeruginosa.

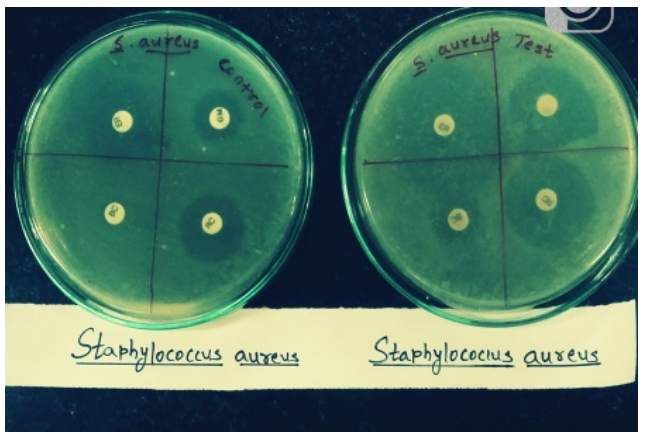

Figure 7: Antibacterial activity of biologically synthesized $1 \mathrm{mM}$ silver nanoparticles coated on antibiotic bacitracin $(10 \mathrm{U})$, Gentamycin $(10 \mathrm{mcg})$, Erythromycin (10 mcg), Ciprofloxacin $(10 \mathrm{mcg})$ against Staphylococcus aureus. against pathogenic microorganisms. This green chemistry approach toward the synthesis of silver nanoparticles has many advantages such as, ease with which the process can be scaled up, economic viability, etc. Applications of such eco-friendly nanoparticles in bactericidal, wound healing and other medical and electronic applications, makes this method potentially exciting for the large-scale synthesis of other inorganic materials. Toxicity studies of silver nanoparticles on human pathogen opens a door for a new range of antibacterial agents.

\section{References}

1. Huang Z, Jiang X, Guo D, Gu N (2011) Controllable synthesis and biomedical applications of silver nanomaterials. J Nanosci Nanotechnol 11: 9395-9408.

2. Prakasha P, Gnanaprakasama P, Emmanuela R, Arokiyarajb S, Saravanan M (2013) Green synthesis of silver nanoparticles from leaf extract of Mimusops elengi, Linn. For enhanced antibacterial activity against multi drug resistant clinical isolates. Colloids Surf B: Biointerfaces 108: 255-259.

3. Sathyavathi R, Krishna MB, Rao SV, Saritha R, Rao DN (2010) Biosynthesis of silver nanoparticles using Coriandrum sativum leaf extract and their application in nonlinear optics. Adv Sci Lett 3:138-143.

4. Awwad AM, Salem NM, Abdeen AO (2013) Green synthesis of silver nanoparticles using carob leaf extract and its antibacterial activity. Int $\mathrm{J}$ Indus Chem 4: 1-6.

5. Bar H, Bhui DK, Sahoo GP, Sarkar P, Priyanka S, et al. (2009) Green synthesis of silver nanoparticles using seed extract of Jatropha curcas. Colloids Surf A Physicochem Eng Asp 348: 212-216.

6. Badr Y, Wahed E, Mahmoud MG (2008) Photo catalytic degradation of methy red dye by silica nanoparticles. J Haz Mat 154: 245-253.

7. Brust M, Walker M, Bethell D, Schiffrin DJ, Whyman R (1994) Synthesis of thio derivatized gold nanoparticles in a two-phase liquid-liquid system. J Chem Soc Chem 801-802.

8. Faure C, Derre A, Neri W (2003) Spontaneous formation of silver nanoparticles in multilamellar vesicles. J Phys Chem B 107: 4515-4526.

9. Janardhanan R, Karuppaiah M, Hebalkar N, Rao TN (2009) Synthesis and surface chemistry of nano silver particles. Polyhedron. 28: 2522-2530.

10. Hussain SM, Hess KL, Gearhart JM, Geiss KT, Schlager JJ (2005) In vitro toxicity of nanoparticles in BRL 3A rat liver cells. Toxicol In Vitro 19: 975-983.

11. Löberg J, Perez Holmberg J, Mattisson I, Arvidsson A, Ahlberg E (2013) Electronic Properties of TiO2 Nanoparticles Films and the Effect on ApatiteForming Ability. Int J Dent.

12. Malarkodi C, Rajeshkumar S, Paulkumar K, Gnanajobitha G, Vanaja M, et al. (2013) Bacterial synthesis of silver nanoparticles by using optimized biomass growth of Bacillus sp. Nanosci Nanotech: An Int J 3: 26-32.

13. Sudha SS, Rajamanickam K, Rengaramanujam J (2013) Microalgae mediated synthesis of silver nanoparticles and their antibacterial activity against pathogenic bacteria. Indian J Exp Biol 51: 393-399. 